Bull. Austral. Math. Soc.

VOL. 53 (1996) [419-424]

\title{
SINGLE IDENTITIES FOR MENDELSOHN AND STEINER 3-QUASIGROUPS
}

\section{ZORAN STOJAKOVIĆ}

\begin{abstract}
We prove that varieties of algebras equivalent to Mendelsohn and Steiner quadruple systems can be defined by single identities.
\end{abstract}

\section{INTRODUCTION}

A Steiner system $S(t, k, v)$ is a pair $(S, T)$, where $S$ is a $v$-set and $T$ is a family of $k$-subsets of $S$ such that every $t$-subset of $S$ is contained in exactly one element of T. An $S(2,3, v)$ is called a Steiner triple system (STS) and an $S(3,4, v)$ is called a Steiner quadruple system (SQS). An ordered analogue of Steiner systems are Mendelsohn systems. A Mendelsohn system $M(t, k, v)$ is a pair $(S, T)$ where $S$ is a $v$-set and $T$ is a family of cyclic $k$-tuples $\left\langle a_{1}, \ldots, a_{k}\right\rangle, a_{1}, \ldots, a_{k}$ distinct elements of $S$, such that every ordered pair of distinct elements from $S$ belongs to exactly one element of $T$. A cyclic $k$-tuple $\left\langle a_{1}, \ldots, a_{k}\right\rangle$ is the following set of $k$ ordered pairs: $\left\langle a_{1}, \ldots, a_{k}\right\rangle=\left\{\left(a_{1}, a_{2}\right),\left(a_{2}, a_{3}\right), \ldots,\left(a_{k-1}, a_{k}\right),\left(a_{k}, a_{1}\right)\right\}$. An $M(2,3, v)$ and an $M(3,4, v)$ are called a Mendelsohn triple system (MTS) and a Mendelsohn quadruple system (MQS), respectively.

It is well known that Steiner and Mendelsohn triple and quadruple systems are equivalent to some algebras. There are two methods of turning STSs into algebras [1, 4].

The first method turns the STS $(S, T)$ into a Steiner quasigroup (squag) $(S, \cdot)$ by defining $x y=z$ if and only if $\{x, y, z\} \in T$ and $x^{2}=x$ otherwise. $(S, \cdot)$ satisfies $x^{2}=x, x y=y x$ and $x(x y)=y$ and every such algebra generates an STS $(S, T)$, where $T$ is the set of 3-element subalgebras of $(S, \cdot)$.

The second method turns the STS $(S, T)$ into a Steiner loop (sloop) $(\bar{S}, \cdot)$, where $\bar{S}=S \cup\{e\}, e \notin S:$ define $x y=z$ if and only if $\{x, y, z\} \in T$ and $x e=e x=x$, $x^{2}=e$ otherwise. $(\bar{S},$.$) satisfies e x=x, x^{2}=e, x y=y x, x(x y)=y$, and, as before, every such algebra generates an STS. We see that the classes of all squags and sloops are varieties.

Analogously, one can obtain similar results for MTSs [6].

Received 26 July 1995

Copyright Clearance Centre, Inc. Serial-fee code: 0004-9729/96 $\$ A 2.00+0.00$. 
By a similar procedure we get that an $\operatorname{SQS}(S, T)$ is equivalent to a 3 -groupoid $(S, f)$ satisfying

$$
\begin{array}{r}
f(f(x, y, z), x, y)=z \\
f(z, y, f(x, y, z))=x \\
f(x, x, y)=y
\end{array}
$$

Every such 3-groupoid is necessarily a 3-quasigroup which is called an SQS-skein [4].

An MQS $(S, T)$ is equivalent to a 3 -groupoid $(S, f)$ satisfying $(1)$ and

$$
f(x, x, y)=f(x, y, x)=y
$$

and this 3-groupoid is also a 3-quasigroup. Such 3-quasigroups we shall call Mendelsohn ternary quasigroups (MTQs).

Besides these bases the varieties of algebras equivalent to SQSs and MQSs can be defined by some other bases which are equivalent to the given ones. For example, it is not difficult to see that identity (1) can be replaced by the equivalent identity

$$
f(x, y, f(z, x, y))=z
$$

\section{Single identities for algebras equivalent to Steiner and MENDELSOHN SYSTEMS}

In [6] it was proved that an MTS $(S, T)$ is equivalent to an algebra $(\bar{S}, \cdot), \bar{S}=$ $S \cup\{e\}, e \notin S$, satisfying $x e=e x=x, x^{2}=e, x(y x)=y$ and also that such algebras can be characterised by a single identity $x(((y y) z) x)=z$. In $[5,6]$ Mendelsohn called systems which are today known as MTSs "generalised Steiner systems" and algebras equivalent to them "generalised Steiner loops and quasigroups". These results of Mendelsohn from [6] were quoted in [4] where the term "sloop" was used instead of "generalised Steiner loop" for the algebra equivalent to an MTS. Such statement is not true, as was shown by Donovan and Oates-Williams in [3].

In [3] it was proved that each of the varieties of sloops and squags can be defined by a single identity. In [2] single identities which define two subvarieties of squags were given.

Now we shall show that algebras equivalent to SQSs and MQSs can be defined by single identities.

The following notation will be used. If $(S, f)$ is a 3-groupoid, then the so-called translation maps $T_{1}(a, b), T_{2}(a, b), T_{3}(a, b)$ can be defined by

$$
T_{1}(y, z)(x)=T_{2}(x, z)(y)=T_{3}(x, y)(z)=f(x, y, z)
$$


THEOREM 1. A 3-groupoid $(S, f)$ is an MTQ if and only if the following identity is satisfied

$$
f(f(x, y, f(u, f(v, v, f(p, q, f(f(z, t, t), p, q))), u)), x, y)=z .
$$

Proof: If $(S, f)$ is an MTQ, then it is easy to see that (6) is satisfied.

Now, let $(S, f)$ be a 3 -groupoid such that (6) is valid. We shall prove that (6) implies (1) and (4).

(6) can be written as:

$$
T_{1}(x, y) T_{3}(x, y) T_{2}(u, u) T_{3}(v, v) T_{3}(p, q) T_{1}(p, q) T_{1}(t, t)=I,
$$

where $I$ is the identity mapping of $S$. From (7) we get that $T_{1}(t, t)$ is $1-1$ and $T_{1}(x, y)$ is onto, hence for all $t \in S, T_{1}(t, t)$ is a bijection, which implies

$$
T_{1}(x, y) T_{3}(x, y) T_{2}(u, u) T_{3}(v, v) T_{3}(p, q) T_{1}(p, q)=T_{1}^{-1}(t, t) .
$$

The last equality implies that $T_{1}(x, y)$ is a bijection and

$$
T_{3}(x, y) T_{2}(u, u) T_{3}(v, v) T_{3}(p, q)=T_{1}^{-1}(x, y) T_{1}^{-1}(t, t) T_{1}^{-1}(p, q) .
$$

By a similar argument we obtain that $T_{3}(x, y)$ is a bijection for all $x, y \in S$, which gives

$$
T_{2}(u, u)=T_{3}^{-1}(x, y) T_{1}^{-1}(x, y) T_{1}^{-1}(t, t) T_{1}^{-1}(p, q) T_{3}^{-1}(p, q) T_{3}^{-1}(v, v) .
$$

Hence $T_{2}(u, u)$ is a bijection for all $u \in S$.

From (7) we get that for all $x, y, u, v, p, q, t, r, s \in S$

$$
\begin{aligned}
& T_{1}(x, y) T_{3}(x, y) T_{2}(u, u) T_{3}(v, v) T_{3}(p, q) T_{1}(p, q) T_{1}(t, t) \\
& \quad=T_{1}(r, s) T_{3}(r, s) T_{2}(u, u) T_{3}(v, v) T_{3}(p, q) T_{1}(p, q) T_{1}(t, t)
\end{aligned}
$$

and

$$
T_{1}(x, y) T_{3}(x, y)=T_{1}(r, s) T_{3}(r, s)
$$

that is,

$$
f(f(x, y, z), x, y)=f(f(r, s, z), r, s) .
$$

By an analogous procedure it follows that for all $x, y \in S$

$$
T_{i}(x, x)=T_{i}(y, y), \quad i=1,2,3,
$$


that is

(9)

$$
f(z, x, x)=f(z, y, y), f(x, z, x)=f(y, z, y), f(x, x, z)=f(y, y, z)
$$

Putting $y=z$ in (9), one gets

$$
f(z, x, x)=f(x, z, x)=f(x, x, z)=f(z, z, z) .
$$

If in (8) we put $x=f(z, z, z), y=r=s=z$, by using (10) it follows that

$$
f(f(f(z, z, z), z, z), f(z, z, z), z)=f(f(z, z, z), z, z)=f(z, f(z, z, z), z)
$$

Since $T_{1}(x, y)$ is a bijection, from the preceding equality we get

$$
f(f(z, z, z), z, z)=z
$$

If now we put $r=s=z$ in (8), it follows that

$$
f(f(x, y, z), x, y)=f(f(z, z, z), z, z)=z
$$

hence identity (1) is valid. So we have proved that for all $x, y \in S, T_{1}(x, y) T_{3}(x, y)=$ I. But identity (1) is equivalent to (5), which means that for all $x, y \in S$, $T_{3}(x, y) T_{1}(x, y)=I$. Therefore, (7) becomes

$$
T_{2}(u, u) T_{3}(v, v) T_{1}(t, t)=I
$$

that is

$$
f(u, f(v, v, f(z, t, t)), u)=z .
$$

Putting $u=v=t=z$ in the preceding identity, and using (10) and (11) we get

$$
f(z, z, z)=z
$$

which by (10) gives

$$
f(z, x, x)=f(x, z, x)=f(x, x, z)=z .
$$

ThEOREM 2. A 3-groupoid $(S, f)$ is an $S Q S$-skein if and only if the following identity is satisfied:

$$
f(f(x, y, f(u, f(v, v, f(p, q, f(f(z, t, t), q, p))), u)), x, y)=z
$$

Proof: If $(S, f)$ is an SQS-skein, then (12) clearly holds. 
Now, let $(S, f)$ be a 3-groupoid such that (12) is satisfied. (12) can be written as

$$
T_{1}(x, y) T_{3}(x, y) T_{2}(u, u) T_{3}(v, v) T_{3}(p, q) T_{1}(q, p) T_{1}(t, t)=I .
$$

By an analogous procedure to that used in the proof of Theorem 1, we get that $T_{1}(x, y)$, $T_{3}(x, y), T_{2}(x, x)$ are bijections for all $x, y \in S$, that

$$
T_{1}(x, y) T_{3}(x, y)=T_{1}(r, s) T_{3}(r, s),
$$

and $f(z, x, x)=f(x, z, x)=f(x, x, z)=f(z, z, z)$. From (14), as before, we get

$$
f(f(x, y, z), x, y)=f(f(z, z, z), z, z)=z,
$$

that is, identity (1) holds.

As in the proof of Theorem 1, we get that $T_{1}(x, y) T_{3}(x, y)=T_{3}(x, y) T_{1}(x, y)=I$. Putting $p=q$ in (13), we get

$$
T_{2}(u, u) T_{3}(v, v) T_{1}(t, t)=I
$$

which implies $f(x, x, z)=z$.

Finally, as in the proof of Theorem 1, we get that

$$
T_{3}(p, q) T_{1}(q, p)=T_{3}(r, s) T_{1}(s, r),
$$

that is,

$$
f(p, q, f(z, q, p))=f(r, s, f(z, s, r)) .
$$

Putting $r=s=z$ in the preceeding equality, we get

$$
f(p, q, f(z, q, p))=z ;
$$

hence identity (2) holds.

\section{REFERENCES}

[1] R.H. Bruck, A survey of binary systems (Springer-Verlag, Berlin, Heidelberg, New York, 1968).

[2] D. Donovan, 'Single laws for two subvarieties of squags', Bull. Austral. Math. Soc. 42 (1990), 157-165.

[3] D. Donovan and S. Oates-Williams, 'Single laws for sloops and squags', Discrete Math. 92 (1991), 79-83.

[4] B. Ganter and H. Werner, 'Co-ordinatizing Steiner systems', in Topics on Steiner Systems, Annals of Discrete Mathematics 7, 1980, pp. 3-24. 
[5] N.S. Mendelsohn, 'A natural generalization of Steiner triple systems', in Computers in Number Theory (Academic Press, New York, 1971), pp. 323-338.

[6] N.S. Mendelsohn, 'A single groupoid identity for Steiner loops', Aequationes Math. 6 (1971), 228-230.

Institute of Mathematics

University of Novi Sad

Trg Dositeja Obradovica 4

2100 Novi Sad

Yugoslavia 